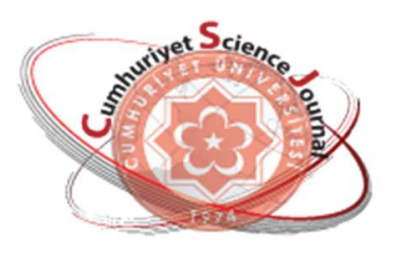

e-ISSN: $2587-246 X$

ISSN : 2587-2680

\section{Cumanoiyet Science Journal \\ esj}

Cumhuriyet Sci. J., Vol.40-1(2019) 108-116

\title{
On Mochizuki-Trooshin Theorem for Sturm-Liouville Operators
}

\author{
İbrahim ADALAR \\ Sivas Cumhuriyet University Zara Veysel Dursun Colleges of Applied Sciences Zara/Sivas, TURKEY \\ Received: 14.10.2018; Accepted: 02.01.2019 \\ http://dx.doi.org/10.17776/csj.470328
}

\begin{abstract}
In this paper, the inverse spectral problems of Sturm-Liouville operators are considered. Some new uniqueness theorems and analogies of the Mochizuki-Trooshin Theorem are proved.

2010 Mathematics Subject Classification. Primary 34A55, 34B24; Secondary 34L05.

Keywords: Inverse spectral problem, Sturm-Liouville equation.
\end{abstract}

\section{Sturm-Liouville Operatörleri için Mochizuki-Trooshin Teoremi Üzerine}

Özet. Bu makalede, Sturm-Liouville operatörlerinin ters spektral problemleri ele alınmıştır. Bazı yeni teklik teoremleri ve Mochizuki-Trooshin teoreminin benzetimleri ispatlanmıştır.

Anahtar Kelimeler: Ters spektral problem, Sturm-Liouville denklemi.

\section{INTRODUCTION}

We consider the classical Sturm-Liouville problem $L=L(q(x), h, H)$

$$
\begin{aligned}
& -y^{\prime \prime}+q(x) y=\lambda y \\
& y^{\prime}(0)-h y(0)=0 \\
& y^{\prime}(1)+H y(1)=0
\end{aligned}
$$

where $h, H \in \mathbb{R}, \lambda$ is a spectral parameter and $q(x) \in L_{1}(0,1)$. The spectrum of such problems consists of countable many real eigenvalues, which have no finite limit point.

The inverse spectral problem for $L$ is to determine the potential function $q(x)$ from some given data. The first result on this area is given by Ambarzumian [1]. Borg [2] showed that generally a single spectrum is insufficient to determine the potential. Levinson [9] showed that if the potential $q(x)$ is symmetric, $q(x)=q(1-x)$, then it is determined uniquely by a single spectrum. Later Gelfand and Levitan [3] proved that the eigenvalues and normalizing coefficients uniquely determine the potential $q(x)$. Hochstadt and Lieberman [7] proved that a single spectra and the potential on the interval $[1 / 2,1]$ uniquely determine the potential $q(x)$ on the whole interval $[0,1]$. 
In 2001, Mochizuki and Trooshin [5] proved a uniqueness theorem for interior spectral data of the SturmLiouville operator. They used similar techniques in [7]. This kind of problems for the Sturm-Liouville operator were formulated and studied in [12-19].

Together with $L$, we consider a boundary value problem $\tilde{L}=L(\tilde{q}(x), h, H)$ of the same form but with a different coefficient $\tilde{q}$. We agree that if a certain symbol $s$ denotes an object related to $L$, then $\tilde{s}$ will denote an analogous object related to $\widetilde{L}$. The eigenvalues and the corresponding eigenfunctions of the problem $L$ are denoted by $\lambda_{n}$ and $\varphi_{n}(x)=\varphi\left(x, \lambda_{n}\right)$, respectively.

The statement of Mochizuki and Trooshin theorem is as following:

Theorem 1.1. [5] If for every $n=0,1,2, \ldots$ we have

$$
\lambda_{n}=\widetilde{\lambda}_{n}, \quad \frac{\varphi_{n}^{\prime}(1 / 2)}{\varphi_{n}(1 / 2)}=\frac{\widetilde{\varphi}_{n}^{\prime}(1 / 2)}{\widetilde{\varphi}_{n}(1 / 2)}
$$

then $q(x)=\tilde{q}(x)$ almost everywhere on $[0,1]$.

The purpose of the present study is to prove some analogies of this theorem and new uniqueness theorems for inverse Sturm-Liouville problems.

In the second section, we give some preliminaries. Section 3 contains new uniqueness theorems and alternative proofs for Mochizuki-Trooshin theorem and Levinson's theorem.

\section{PRELIMINARIES}

We shall first mention some known results which will be needed later. Let $\varphi(x, \lambda)$ be the solution of equation (1) satisfying the initial conditions,

$$
\varphi(0, \lambda)=1, \varphi^{\prime}(0, \lambda)=h .
$$

We need specifically to focus on the properties of $\varphi(1 / 2, \lambda)$. It is known that, $[4,8,17,18]$ for each $x \in[0,1], \varphi(x, \lambda)$ and $\varphi^{\prime}(x, \lambda)$ are entire functions of $\lambda$ and there exist some constants $c_{1}, c_{2}>0$ such that $\varphi(1 / 2, \lambda)$ and $\varphi^{\prime}(1 / 2, \lambda)$ are all bounded by $c_{1} \exp \left(c_{2}|\lambda|^{1 / 2}\right)$. For $|\lambda| \rightarrow \infty$ uniformly with respect to $x \in[0,1]$,

$$
\begin{aligned}
& \varphi(x, \lambda)=\cos \rho x+O\left(\frac{\exp \tau x}{\rho}\right) \\
& \varphi^{\prime}(x, \lambda)=-\rho \sin \rho x+O(\exp \tau x) .
\end{aligned}
$$

Here $\rho=\sqrt{\lambda}$ and $\tau=|\operatorname{Im} \rho|$. The function

$$
\omega(\lambda)=\varphi^{\prime}(1, \lambda)+H \varphi(1, \lambda)
$$

is entire in $\lambda$ and it has an at most countable set of zeros, $\left\{\lambda_{n}\right\}$. Denote

$$
G_{\delta}=\{\rho:|\rho-k \pi| \geq \delta, k=0, \pm 1, \pm 2, \ldots\}, \delta>0 .
$$

We have that [8] 


$$
|\omega(\lambda)| \geq C_{\delta}|\rho| \exp \tau
$$

for $\rho \in G_{\delta},|\rho| \geq \rho^{*}$ and sufficiently large $\rho^{*}$. The Weyl $m_{-}$function is defined by:

$$
m_{-}(a, \lambda)=-\frac{\varphi(a, \lambda)}{\varphi^{\prime}(a, \lambda)}
$$

where $a \in[0,1]$. The following Marchenko's uniqueness theorem [6] is also necessary

for our analysis.

Theorem 2.1. [6] The Weyl $m_{-}(a, \lambda)$ function uniquely determines $h$ as well as $q(x)$ almost everywhere on $[0, a]$.

\section{UNIQUENESS THEOREMS}

Here we provide an alternative proof for Mochizuki and Trooshin theorem.

Proof of the Theorem 1.1. Consider the initial-value problems:

$$
\begin{aligned}
& -\varphi^{\prime \prime}+q(x) \varphi=\lambda \varphi \\
& \varphi(0)=1, \varphi^{\prime}(0)=h
\end{aligned}
$$

and

$$
\begin{aligned}
& -\tilde{\varphi} "+\tilde{q}(x) \tilde{\varphi}=\lambda \tilde{\varphi} \\
& \tilde{\varphi}(0)=1, \tilde{\varphi}^{\prime}(0)=h .
\end{aligned}
$$

The functions $\varphi(x, \lambda)$ and $\varphi^{\prime}(x, \lambda)$ satisfy

$$
\tilde{\varphi}(0, \lambda) \varphi^{\prime}(0, \lambda)-\varphi(0, \lambda) \tilde{\varphi}^{\prime}(0, \lambda)=0 .
$$

Multiplying (8) by $\tilde{\varphi}(x, \lambda)$ and (9) by $\varphi(x, \lambda)$, subtracting, and integrating from 0 to $1 / 2$, we obtain

$$
f(\lambda)=\int_{0}^{1 / 2}(q(x)-\tilde{q}(x)) \varphi(x, \lambda) \tilde{\varphi}(x, \lambda) d x=\tilde{\varphi}(1 / 2, \lambda) \varphi^{\prime}(1 / 2, \lambda)-\varphi(1 / 2, \lambda) \tilde{\varphi}^{\prime}(1 / 2, \lambda) .
$$

The conditions of the theorem imply

$$
f\left(\lambda_{n}\right)=0
$$

Define $h(\lambda)=\frac{f(\lambda)}{\omega(\lambda)}$, which is an entire function. From the asymptotics (6) and (7) for $f(\lambda)$ and $\omega(\lambda)$, we see that

$$
h(\lambda)=O\left(\frac{1}{|\rho|}\right)
$$

for large $|\rho|$. Thus, by Liouville's theorem, we obtain for all $\lambda$, 


$$
h(\lambda)=0
$$

or

$$
f(\lambda)=0
$$

From (10), we have that

$$
\frac{\varphi(1 / 2, \lambda)}{\varphi^{\prime}(1 / 2, \lambda)}=\frac{\tilde{\varphi}(1 / 2, \lambda)}{\tilde{\varphi}^{\prime}(1 / 2, \lambda)}
$$

and hence

$$
m_{-}(1 / 2, \lambda)=\widetilde{m_{-}}(1 / 2, \lambda) \text {. }
$$

By Theorem 2.1, we prove $q(x)=\tilde{q}(x)$ almost everywhere on $[0,1 / 2]$.

To prove that $q(x)=\tilde{q}(x)$ almost everywhere on $[1 / 2,1]$, we will consider the supplementary problem $\bar{L}$ :

$$
\begin{gathered}
-y^{\prime \prime}+q(1-x) y=\lambda y \\
y^{\prime}(0)-H y(0)=0 \\
y^{\prime}(1)+h y(1)=0 .
\end{gathered}
$$

Since $\varphi_{n}(1-x)=\bar{\varphi}_{n}(x)$, the assumption conditions in Theorem 1.1 are still satisfied. If we repeat the above arguments then this yields $q(1-x)=\tilde{q}(1-x)$ on $[0,1 / 2]$, that is $q(x)=\tilde{q}(x)$ almost everywhere on $[1 / 2,1]$. This completes the proof.

By the remark to proof of Theorem 1, we have proved the following result:

Corollary 3.1. Let $f(\lambda)=0$ for all $\lambda$. If for every $n=0,1,2, \ldots$ we have

$\lambda_{n}=\widetilde{\lambda}_{n}$

then $q(x)=\tilde{q}(x)$ almost everywhere on $[0,1]$.

Let $L_{0}$ :

$-y^{\prime \prime}+q(x) y=\lambda y$

$y^{\prime}(0)-h y(0)=0$

$y^{\prime}(1)+h y(1)=0$.

Here we provide an alternative proof for the following Levinson's theorem [9].

Theorem 3.2. [9] If $q(x)=q(1-x)$ then the function $q(x)$ and $h$ are uniquely determined by the spectrum of problem $L_{0}$. 
Proof. Applying the same arguments as that in the proof of Theorem 1.1, we can see that

$$
f(\lambda)=2 \int_{0}^{1 / 2}(q(x)-\tilde{q}(x)) \varphi(x, \lambda) \tilde{\varphi}(x, \lambda) d x=0
$$

and hence

$$
f\left(\lambda_{n}\right)=2 \int_{0}^{1 / 2}(q(x)-\tilde{q}(x)) \varphi\left(x, \lambda_{n}\right) \tilde{\varphi}\left(x, \lambda_{n}\right) d x=0 .
$$

We obtain for all $\lambda$,

$$
f(\lambda)=\tilde{\varphi}(1 / 2, \lambda) \varphi^{\prime}(1 / 2, \lambda)-\varphi(1 / 2, \lambda) \tilde{\varphi}^{\prime}(1 / 2, \lambda)=0
$$

Thus we arrive at

$$
m_{-}(1 / 2, \lambda)=\widetilde{m_{-}}(1 / 2, \lambda)
$$

By Theorem 2.1, the proof is complete.

Let us consider the following Sturm-Liouville problems

$$
\begin{aligned}
& -y^{\prime \prime}+q(x) y=\lambda y \\
& y(0)=y(1 / 2)=0 \\
& y(0)=y^{\prime}(1 / 2)=0 .
\end{aligned}
$$

Let $\left\{\mu_{n}\right\}_{n=0}^{\infty}$ and $\left\{v_{n}\right\}_{n=0}^{\infty}$ be the spectra of the problems (11), (12) and (11), (13), respectively. Consider the problem: given three spectra $\left\{\lambda_{n}\right\}_{n=0}^{\infty},\left\{\mu_{n}\right\}_{n=0}^{\infty}$ and $\left\{v_{n}\right\}_{n=0}^{\infty}$ determine $q(x)$. Knowledge of $\left\{\mu_{n}\right\}_{n=0}^{\infty}$ and $\left\{v_{n}\right\}_{n=0}^{\infty}$ is equivalent to the knowledge of $q(x)$ on $[0,1 / 2]$. Thus this problem is the HochstadtLieberman problem in [7]. Now consider the problem: given $\left\{\lambda_{n}\right\}_{n=0}^{\infty} \subset\left\{\left\{v_{n}\right\}_{n=0}^{\infty} \cup\left\{\mu_{n}\right\}_{n=0}^{\infty}\right\}$ determine $q(x)$. In this case, only spectra $\left\{\lambda_{n}\right\}_{n=0}^{\infty}$ uniquely determine the potential $q(x)$ on the whole $[0,1]$. We can give the following uniqueness theorem.

Theorem 3.3. Let $\left\{\lambda_{n}\right\}_{n=0}^{\infty} \subset\left\{\left\{v_{n}\right\}_{n=0}^{\infty} \cup\left\{\mu_{n}\right\}_{n=0}^{\infty}\right\}$ and $\left\{\widetilde{\lambda}_{n}\right\}_{n=0}^{\infty} \subset\left\{\left\{\widetilde{v}_{n}\right\}_{n=0}^{\infty} \cup\left\{\widetilde{\mu}_{n}\right\}_{n=0}^{\infty}\right\}$. If for every $n=0,1, \ldots$ we have

$\lambda_{n}=\widetilde{\lambda}_{n}$,

then $q(x)=\tilde{q}(x)$ almost everywhere on $[0,1]$.

Proof. As in the proof of Theorem 1.1, we can show that $f(\lambda)=\int_{0}^{1 / 2}(q(x)-\tilde{q}(x)) \varphi(x, \lambda) \tilde{\varphi}(x, \lambda) d x=\tilde{\varphi}(1 / 2, \lambda) \varphi^{\prime}(1 / 2, \lambda)-\varphi(1 / 2, \lambda) \tilde{\varphi}^{\prime}(1 / 2, \lambda)$.

To prove, as in the Corollary 3.1, it suffices to show that $f(\lambda)=0$ for all $\lambda$. The assumptions of the theorem imply that

$$
\varphi_{n}\left(1 / 2, \lambda_{n}\right)=0 \text { or } \varphi_{n}^{\prime}\left(1 / 2, \lambda_{n}\right)=0 \text { and } \tilde{\varphi}_{n}\left(1 / 2, \lambda_{n}\right)=0 \text { or } \tilde{\varphi}_{n}^{\prime}\left(1 / 2, \lambda_{n}\right)=0 \text {. }
$$


Hence, we have

$$
f\left(\lambda_{n}\right)=0
$$

Thus, repeating the proof Theorem 1.1, we arrive at

$$
f(\lambda)=0,
$$

which implies that

$$
m_{-}(1 / 2, \lambda)=\widetilde{m_{-}}(1 / 2, \lambda)
$$

and $q(x)=\tilde{q}(x)$ almost everywhere on $[0,1 / 2]$. The supplementary problem $\bar{L}$ in proof of Theorem 1.1 completes the proof.

Let us define

$$
g(\rho)=\int_{0}^{1 / 2}(q(x)-\tilde{q}(x)) \varphi(x, \lambda) \tilde{\varphi}(x, \lambda) d x=\tilde{\varphi}(1 / 2, \lambda) \varphi^{\prime}(1 / 2, \lambda)-\varphi(1 / 2, \lambda) \tilde{\varphi}^{\prime}(1 / 2, \lambda)
$$

where $\rho=\sqrt{\lambda}$. The asymptotics (6) imply that the entire function $g(\rho)$ is a function of exponential type $\leq 1$. As shown by the above discussion, let $g(\rho)=0$ then only spectra $\left\{\lambda_{n}\right\}_{n=0}^{\infty}$ uniquely determine the potential $q(x)$ on $[0,1]$. We now consider the problem: If the zeros of an entire function of exponential type are known to include a given sequence of positive real numbers what can be said about growth of the function. The first result of this type is given by Carlson's Theorem. This theorem [11, p.153] says, if $g$ is entire function of exponential type $<\pi$ and vanishes on the positive integers then $g$ vanishes everywhere. This

idea has been further developed by Rubel [10, p.422]:

Theorem 3.4. [10] Let $\rho=t+i \tau$ and $\Omega=\left\{\rho_{n}: \rho_{n+1}-\rho_{n} \geq \gamma>0, \rho_{n}>0, n \in \mathbb{Z}^{+}\right\}$. In order to each entire function $g(\rho)$ satisfying

$$
\begin{aligned}
& g(\rho)=O(1) \exp (a|\rho|), \quad a<\infty \\
& g(i \tau)=O(1) \exp (b|\tau|), \quad b<\delta \\
& g\left(\rho_{n}\right)=0
\end{aligned}
$$

vanish identically, it is sufficient that

$$
\inf _{\rho>1} \lim _{k \rightarrow \infty} \sup (\ln \rho)^{-1} \sum_{\rho_{n} \leq p k} \frac{1}{\rho_{n}}=L(\Omega) \geq \frac{\delta}{\pi}
$$

Here, $L(\Omega)$ is the logarithmic block density of $\Omega$.

We turn repeat that equation (14). From asymptotics (6), the entire function

$$
g(\rho)=\int_{0}^{1 / 2}(q(x)-\tilde{q}(x)) \varphi(x, \lambda) \tilde{\varphi}(x, \lambda) d x
$$


satisfies (15) and (16). Also we have that

$\rho_{n+1}-\rho_{n}>0$

where $\sqrt{\lambda_{n}}=\rho_{n}$. In this case, we can give a uniqueness theorem by using Theorem 3.4.

Theorem 3.5. Let $\Lambda \subset \mathbb{N} \cup\{0\}$ be a subset of nonnegative integer numbers and let $\Omega:=\left\{\lambda_{n}\right\}_{n \in \Lambda}$ be a part of the spectrum of $L$ such that the numbers $\sqrt{\lambda_{n}}=\rho_{n}$ satisfy (18) for function $g(\rho)$. If for $n \in \Lambda$, we have

$\lambda_{n}=\widetilde{\lambda}_{n}, \quad \frac{\varphi_{n}^{\prime}(1 / 2)}{\varphi_{n}(1 / 2)}=\frac{\widetilde{\varphi}_{n}^{\prime}(1 / 2)}{\widetilde{\varphi}_{n}(1 / 2)}$

then $q(x)=\tilde{q}(x)$ almost everywhere on $[0,1]$.

Proof. As in the proof of Theorem 1, we obtain

$g(\rho)=\int_{0}^{1 / 2}(q(x)-\tilde{q}(x)) \varphi(x, \lambda) \tilde{\varphi}(x, \lambda) d x=\tilde{\varphi}(1 / 2, \lambda) \varphi^{\prime}(1 / 2, \lambda)-\varphi(1 / 2, \lambda) \tilde{\varphi}^{\prime}(1 / 2, \lambda)$.

The assumptions of the theorem imply

$g\left(\rho_{n}\right)=0, n \in \Lambda$.

By the Theorem 3.4, we have that

$g(\rho)=0$

on the whole $\rho$-plane. Thus, $\varphi(x, \lambda)$ and $\tilde{\varphi}(x, \lambda)$ satisfy

$\tilde{\varphi}(1 / 2, \lambda) \varphi^{\prime}(1 / 2, \lambda)-\varphi(1 / 2, \lambda) \tilde{\varphi}^{\prime}(1 / 2, \lambda)=0$

and hence

$m_{-}(1 / 2, \lambda)=\widetilde{m_{-}}(1 / 2, \lambda)$.

By the Theorem 2.1, we prove $q(x)=\tilde{q}(x)$ almost everywhere on [0,1/2]. Repeating the supplementary problem in the last part of proof of Theorem 1.1, we can show that $g(\rho)=0$ on the whole $\rho$-plane, which implies that $q(x)=\tilde{q}(x)$ on $[1 / 2,1]$ and consequently, $q(x)=\tilde{q}(x)$ almost everywhere on $[0,1]$. This completes the proof.

Let us consider the Sturm-Liouville problem $L$ for $q(x) \in L_{2}(0,1)$. Horvath [15, 19, p.268] proved Hochstadt-Lieberman type an uniqueness theorem by using simple closedness properties of the exponential system corresponding to the known eigenvalues. We can give the following uniqueness theorem with same arguments in [15] for Mochizuki-Trooshin type theorem. 
Theorem 3.6. Let $\Lambda \subset \mathbb{N} \cup\{0\}$ be a subset of nonnegative integer numbers and let $\Omega:=\left\{\lambda_{n}\right\}_{n \in \Lambda}$ be a part of the spectrum of $L$ such that the system of functions $\left\{\cos 2 \rho_{n} x\right\}_{n \in \Lambda}$ is complete in $L_{2}(0,1 / 2)$. If for $n \in \Lambda$, we have

$$
\lambda_{n}=\widetilde{\lambda}_{n}, \quad \frac{\varphi_{n}^{\prime}(1 / 2)}{\varphi_{n}(1 / 2)}=\frac{\widetilde{\varphi}_{n}^{\prime}(1 / 2)}{\widetilde{\varphi}_{n}(1 / 2)}
$$

then $q(x)=\tilde{q}(x)$ almost everywhere on $[0,1]$.

Proof. As in the proof of Theorem 1, we can show that

$$
f(\lambda)=\int_{0}^{1 / 2}(q(x)-\tilde{q}(x)) \varphi(x, \lambda) \tilde{\varphi}(x, \lambda) d x=\tilde{\varphi}(1 / 2, \lambda) \varphi^{\prime}(1 / 2, \lambda)-\varphi(1 / 2, \lambda) \tilde{\varphi}^{\prime}(1 / 2, \lambda) .
$$

Hence, we have that

$$
f\left(\lambda_{n}\right)=0, n \in \Lambda
$$

The following representation holds $[4,6,8]$

$$
\varphi(x, \lambda)=\cos \rho x+\int_{0}^{x} K(x, t) \cos \rho t d t
$$

where $K(x, t)$ is a continuous function which does not depend on $\lambda$. Hence,

$$
\varphi(x, \lambda) \tilde{\varphi}(x, \lambda)=\frac{1}{2}\left(1+\cos 2 \rho x+\int_{0}^{x} K_{1}(x, t) \cos \rho t d t\right)
$$

where $K_{1}(x, t)$ is a continuous function which does not depend on $\lambda$. From (19) and (20), we have

$$
\int_{0}^{1 / 2}\left[\phi(x)+\int_{x}^{1 / 2} K_{1}(x, t) \phi(t) d t\right] \cos 2 \rho_{n} x d x+\int_{0}^{1 / 2} \phi(x) d x=0, n \in \Lambda,
$$

where $\phi(x)=q(x)-\tilde{q}(x)$. By the Riemann-Lebesgue lemma,

$$
\int_{0}^{1 / 2} \phi(x) d x=0
$$

By the completeness of the functions $\left\{\cos 2 \rho_{n} x\right\}_{n \in \Lambda}$ we have

$$
\phi(x)+\int_{x}^{1 / 2} K_{1}(x, t) \phi(t) d t=0 .
$$


Since this homogeneous integral equation has only the trivial solution it follows that and $q(x)=\tilde{q}(x)$ almost everywhere on $[0,1 / 2]$. The supplementary problem $\bar{L}$ in proof of Theorem 1.1 completes the proof.

\section{REFERENCES}

[1]. Ambarzumyan, V.A., Uber eine Frage der Eigenwerttheorie, Z. Phys. 53 (1929) 690-695.

[2]. Borg, G., Eine umkehrung der Sturm-Liouvillesehen eigenwertaufgabe, Acta Math. 78 (1946) 1-96.

[3]. Gelfand, L.M., Levitan, B.M., On the determination of a differential equation from its spectral function, Izv. Akad. Nauk SSR. Ser. Mat. 15 (1951) 309-360 (in Russian), English transl. in Amer. Math. Soc.. Transl. Ser. 2 (1) (1955) 253-304.

[4]. Levitan B.M., Sargsjan I.S., Sturm-Liouville and Dirac Operators. Dordrecht: Kluwer; 1991.

[5]. Mochizuki, K., Trooshin, I., Inverse problem for interior spectral data of Sturm-Liouville operator, J. Inverse Ill-posed Probl. 9 (2001) 425-433.

[6]. Marchenko V., Some questions in the theory of one-dimensional linear differential operators of the second order. I. Tr.Mosk. Mat. Obs. (1952) 1:327-420. (Russian). English transl. in Am.Math. Soc. Trans. (1973) 2:1-104.

[7]. Hochstadt, H., Lieberman, B., An inverse Sturm-Liouville problem with mixed given data, SIAM J. Appl. Math. 34 (1978) 676-680.

[8]. Freiling, G., Yurko, V.A., Inverse Sturm-Liouville Problems and Their Applications, NOVA Science Publishers, New York, 2001.

[9]. Levinson, N., The inverse Sturm-Liouville problem, Math. Tidsskr, 13 (1949), 25- 30.

[10].Rubel, L.A., Necessary and suffcient conditions for Carison's theorem on entire functions, Trans. Amer. Math. Soc. vol. 83 (1956) 417-429.

[11].Boas, R.P., Entire functions, New York, Academic Press, 1954.

[12].Mochizuki, K., Trooshin, I., Inverse problem for interior spectral data of the Dirac operator on a finite interval, Publ. RIMS, Kyoto Univ. 38 (2002) 387-395.

[13]. Sat, M., Panakhov, E., A uniqueness theorem for Bessel operator from interior spectral data. Abstr. Appl. Anal., Volume 2013, Article ID 713654, 6 pages.

[14].Ozkan, A.S., Amirov, R. Kh., An interior inverse problem for the impulsive Dirac operator, Tamkang Journal of Mathematics, 42 (2011) 259-263.

[15].Horvath, M., Inverse spectral problems and closed exponential systems, Ann. of Math. 162 (2005) 885-918.

[16].Panakhov, E., Sat, M., Inverse problem for the interior spectral data of the equation of hydrogen atom, Ukrainian Mathematical Journal, 64 (2013), no.11.

[17].Guo, Y., Wei, G., Inverse Sturm-Liouville problems with the potential known on an interior subinterval, Appl. Anal., 94 (5) (2015) 1025-1031.

[18].Gesztesy F., Simon B., Inverse spectral analysis with partial information on the potential. II. The case of discrete spectrum. Trans. Am. Math. Soc. 352 (6) (2000) 2765-2787.

[19].Shieh, C.T., Yurko, V.A., Inverse nodal and inverse spectral problems for discontinuous boundary value problems. J. Math. Anal. Appl. 347 (2008) 266-272. 